\title{
De La Politique De Santé Publique Aux Démarches D’entraide Sociale : Mutuelles De Santé Et Équité Sociale Dans Le Département Du Borgou Au Bénin
}

\author{
Yvette Onibon Doubogan \\ Sociologue, Maître Assistante des Universités (CAMES), \\ Enseignante chercheure à l’Université de Parakou, Bénin
}

Agnès Oladoun Badou Savi,

Socio-anthropologue, Assistante à l'Ecole Supérieure des Assistants Sociaux de la Faculté des Sciences de la Santé, Université d’Abomey-Calavi, Bénin

\section{Roger A. Atchouta}

Doctorant en Sociologie du développement,

Université d'Abomey-Calavi, Bénin

doi: 10.19044/esj.2016.v13n3p134 URL:http://dx.doi.org/10.19044/esj.2016.v13n3p134

\begin{abstract}
Apart from its impact on the improvement of health care system, the mutual health insurance implies some changes on the management of health care at family level. This study analyzes the role played by mutual health insurance in the promotion of social equity within families in the Department of Borgou (North -east Benin). It also focuses on qualitative research methodology which combines documentary analysis, life experiences recounting and direct observation for data collection. The empirical corpus triangulation based on the theoretical perspective to social reinforcement of gender roles from Vidal (2008) reveals that the operationalization of insurance systems in Benin cotton basin which has contributed to a redefinition of gender roles in terms of family health care. At the event of mutual health insurances, the responsibilities in terms of family health care formerly devoted exclusively to women, are highly in a movement and then, are shared between wife and husband inner the couple. The mutual health insurance systems affect the private sphere and have influenced the existing relationship between men and women on the health care where, men have to play an important role towards family health care.
\end{abstract}

Keywords: Mutual health insurances, gender role, equity, family health care, health care systems 


\section{Résumé}

Les mutuelles de santé au-delà de leur impact sur l'amélioration du système de santé, engendrent des transformations dans la gestion de la santé au niveau familial. Cette recherche analyse la place des mutuelles de santé dans la promotion de l'équité sociale au sein des familles dans le département du Borgou au nord-est du Bénin. Elle s'appuie sur une démarche méthodologique qualitative qui combine pour la collecte des informations, l'analyse documentaire, les récits de vie et l'observation directe. La triangulation du corpus empiriques dans la perspective théorique de la construction sociale des rôles du genre de Vidal (2008) révèle que l'opérationnalisation des mutualités dans le bassin cotonnier du Bénin a contribué à une redéfinition des rôles de genre en matière de gestion des soins de santé familiale. Avec l'avènement des mutuelles de santé, les rôles en matière de santé familiale antérieurement dévolus exclusivement aux femmes, sont en pleine mutation et sont désormais partagés entre homme et femme au sein du couple. Les mutuelles de santé entrent dans la sphère privée et transforment les rapports hommes et femmes autour de la santé où les hommes prennent de plus en plus une place prépondérante dans les soins de santé familiale.

Mots clés : Mutuelles de santé, rôle de genre, équité, santé familiale, politiques de santé, Département du Borgou (Bénin)

\section{Introduction}

Les politiques de santé publique mise en œuvre au Bénin depuis de longues années n'ont jusque-là pas permis d'atteindre les Objectifs du Millénaire pour le Développement (OMD) en matière de santé, notamment l'objectif $n^{\circ} 5$. La majorité de la population peine encore à accéder aux soins de santé. Les disparités d'accès aux soins de santé sont toujours prégnantes. D'ailleurs, le système de protection sociale mis en place par l'Etat béninois (sécurité sociale et assistance sociale) ne concerne à peine que 15 à $20 \%$ de la population (PNDS, 2011). Les trois obstacles à l'accès aux soins des populations (géographique, financier et socio-culturel) demeurent toujours vivaces selon les travaux de Doumbouya (2008). En effet, le coût d'accès élevé aux soins de santé modernes combiné à la pauvreté des ménages constitue un facteur majeur à l'accessibilité aux soins. De fait, les inégalités d’accès aux soins et services de santé pour les ménages constituent un problème central en matière de santé publique. C'est dans un tel contexte marqué par le désengagement de l'Etat et un accès différentiel aux soins et services de santé pour les populations en fonction du niveau de protection socio-sanitaire que les mutuelles de santé comme un mode de financement alternatif au système de soins se sont imposées. 
Calquées sur la mutualité européenne et réadaptée à la solidarité traditionnelle africaine, les mutuelles de santé ont fait leur apparition en Afrique subsaharienne à la suite de l'Initiative d'Alma-Ata de 1978 et celle de Bamako 1987. Les mutuelles de santé, plus précisément les assurances santé communautaires à but non lucratif sont des organisations communautaires qui visent à améliorer l'accès aux soins de la population par le partage des risques maladie entre les membres (Waelkens et Criel, 2004). Ce système d'assurance santé est une forme de solidarité entre citoyens dans le domaine de la santé faisant supporter les charges de soins des "mal portant" par les "bien portant". La mutuelle de santé devient ainsi une réponse adaptée à la protection sociale des couches vulnérables, en permettant à toute personne dans le besoin, d'accéder à des soins de santé de qualité quel que soit son niveau de revenus. Le Projet Mutuelles de Santé intervenant dans le Nord-Bénin depuis 1994 avec l'appui financier de la coopération suisse s'est inscrit dans cette logique de mutualisation solidaire et communautaire en matière de prévoyance de la santé.

La problématique générale de cet article trouve sa pertinence dans l'analyse du financement des dépenses de santé au sein des familles. Dans un contexte de rareté des ressources, de monétarisation de l'accès aux soins et de responsabilisation des femmes quant à la prise en charge des soins de santé, les mutuelles opèrent des transformations et des remises en causes des rôles sociaux attribués à chaque sexe. S’il a été prouvé que le fonctionnement des mutuelles de santé augmente sensiblement l'accès aux soins et l'amélioration de la qualité de ces derniers, il est important de questionner la dimension de l'influence des mutuelles de santé sur l'un de ses objectifs de départ : l'équité sociale. Plus précisément il s’agit de comprendre dans quelle mesure les mutuelles de santé permettent non seulement à toutes les couches sociales de bénéficier de soins de qualité mais aussi induisent une nouvelle configuration des rôles sociaux de genre en matière de santé. Dans les communautés Bariba et Peulh du Borgou, la répartition traditionnelle des rôles confère aux femmes les responsabilités de prévention et des premiers soins aux membres de la famille. Ainsi, il revenait aux femmes seules d'investir et de rechercher selon les ressources dont-elles disposent, des types de soins aux membres de la famille. Il leur appartient également de faire face seules aux tracasseries administratives dans le processus de recours aux soins dans les centres de santé. Dans ce processus de division et de répartition sexuelle des rôles sociaux, comment la mutuelle de santé, en tant que structure d'économie sociale peut-elle constituer une alternative durable à l'équité et à l'égalité entre les femmes et les hommes en matière de gestion financière de la santé familiale ? Comment les mutuelles de santé parviennent-elles à déconstruire la répartition des rôles sociaux existants en matière de santé? Dans un angle plus large, comment un 
dispositif de promotion de l'accessibilité financière des populations devient un outil transformateur des relations du genre au sein des familles ? Ce sont les réponses à ces questionnements qui constituent la trame de cet article.

\section{Matériel et méthodes}

Cette recherche adopte une démarche méthodologique essentiellement qualitative, car elle s'inscrit dans la logique de saisir la dynamique fonctionnelle des mutuelles de santé. Elle vise également à mettre en évidence l'influence des mutuelles de santé sur l'organisation familiale des dépenses de santé et son implication sur les rôles de genre en matière de santé dans le département du Borgou.

Elle a mobilisé pour le recueil des données la recherche documentaire, l'observation directe, les récits de vie, les entretiens individuels approfondis. La recherche documentaire a consisté en la recension et en l'analyse des documents qui mettent en exergue la capitalisation des expériences du Projet de Mutuelles de Santé mis en œuvre par la Coopération Suisse avec un accent particulier sur son impact sur la santé familiale et communautaire et sa stratégie d'entraide sociale et de cohésion familiale. Elle a notamment permis de disposer d'informations sur le contexte qui a favorisé la mise en place des mutuelles de santé au Bénin, les mécanismes d'assurance maladie communautaire, ainsi que l'influence des mutuelles de santé sur les rôles sociaux de genre en matière de santé. L'observation directe a eu pour avantage de permettre d'observer l'attitude de certains mutualistes en situation de fréquentation des centres de santé. Quant aux récits de vie, ils ont permis de saisir les expériences de quelques personnes en matière de changements sociaux induits par la mise en place des mutuelles de santé dans leur localité. Les entretiens individuels approfondis servi à renseigner sur le mode de fonctionnement des mutuelles de santé, l'implication des mutuelles sur la protection sociale de même que la manière dont les mutuelles de santé contribuent à réduire les inégalités sociales en matière d'accès aux soins de santé. A cet effet, 30 entretiens individuels approfondis ont été réalisés avec les mutualistes, les non mutualistes, les gestionnaires de mutuelles de santé, des leaders communautaires, des élus locaux et des agents de santé. Ces différents acteurs ont été sélectionnés par choix raisonné dans les communes de Nikki, Kalalé, Pèrèrè. A l'intérieur de ces communes, les localités de Blémi, Gbassi et Gnelbotché (à Kalalé), de Gah Gbérou et Fodarou (à Nikki) et de Bonrou (à Pèrèrè) ont été approchées compte tenu du fait qu'elles abritent des mutuelles de santé.

L'analyse de contenu thématique a permis le traitement des données documentaires et empiriques. En effet, il a été procédé à une catégorisation des informations recueillies suivant les principaux axes de la recherche. Les 
différentes données ainsi constituées ont été croisées puis analysées dans la perspective holistique du genre et de santé. La triangulation des données a permis de mettre en évidence la compréhension des rôles sociaux et de l'équité sur l'opérationnalisation des politiques d'entraide sociale en matière de santé familiale. L’approche théorique de fond qui a gouverné la collecte et l'analyse des données est celle de la construction sociale des rôles du genre (Vidal, 2008).

\section{Résultats}

Bien que les résultats soient focalisés sur les mutuelles de santé comme instrument de transformation des rôles de genre et de l'équité sociale, une attention particulière a été accordée également à l'historique et à d'autres effets induits par ces structures d'économie sociale.

\section{Analyse diachronique et descriptive du mode de fonctionnement des mutuelles de santé dans le Nord-Est Bénin}

Les premières mutuelles de santé sont apparues au Bénin dans les années 1990. Mais ce n'est qu'en 1994, qu'elles ont été introduites dans le département du Borgou, grâce au Centre International de Développement et de Recherche (CIDR) avec l'appui financier de la Coopération Suisse à travers un Projet dénommé «Projet Mutuelles de santé » aujourd'hui « Appui Suisse aux Mutuelles de Santé ». L'objectif visé par la création de ces mutuelles de santé était de faciliter l'accessibilité financière des populations rurales pauvres et défavorisées à des soins et services de santé de qualité. En effet, en dépit de l'existence d'hôpitaux privés sociaux fonctionnels à Bembéréké, Boko et Nikki, il était observé une exclusion financière marquée par la faible fréquentation des communautés locales des services hospitaliers de ces différents centres de santé. C'est donc pour offrir une égalité de chance aux populations dans l'accès aux soins de santé que les mutuelles de santé ont été mise en place.

Si les expériences des mutuelles de santé sont relativement récentes au Bénin, et plus particulièrement dans le département du Borgou, leur émergence s’est accélérée au cours des dix dernières années. Par exemple, seules 11 mutuelles de santé ont été dénombrées sur l’ensemble du pays en 1997. Aujourd'hui, on dénombre au total trois cent treize (313) mutuelles réparties sur l'ensemble du territoire national, dont 31 dans le département du Borgou. Malgré la jeunesse du mouvement mutualiste au Bénin, des réseaux ont commencé à se structurer dans certaines parties du pays, en tant que composantes opérationnelles de promotion de la viabilité des mutuelles de santé. Ainsi, dans le département du Borgou, il y a eu la création du Réseau Alliance Santé (RAS), les Unions Communales des Mutuelles de Santé de Bembéréké, de Sinendé et de Banikoara. A titre d'exemple, le 
Réseau Alliance Santé créé en Octobre 2002 regroupe en 2016, 22 mutuelles de santé qui couvrent environ 17000 assurés mutualistes reparties dans les communes de Nikki, Pèrèrè, N’Dali, Parakou et Tchaourou (Aladji Boni Sylla, nd).

Les organes qui composent les mutuelles de santé sont : l'Assemblée Générale, le Conseil d’Administration, le Bureau Exécutif et le Contrôle Interne. L'adhésion à la mutuelle de santé se fait à travers un groupement mutualiste. Le groupement mutualiste est composé des ménages qui se font confiance et se mettent ensemble pour trouver des solutions à leurs problèmes de santé. L'ensemble des ménages composent l'Assemblée Générale. C'est l'organe suprême de décision de la mutuelle de santé. Par contre, le Conseil d'Administration des mutuelles est l'organe opérationnel qui définit les actions de la mutuelle de santé. Il est formé des représentants de chaque groupement mutualiste. Afin d'exécuter ses décisions, le conseil élit en son sein un bureau exécutif de trois membres et un Contrôleur Interne. Le Bureau de la mutuelle de santé est composé de trois personnes élues par le Conseil d'Administration en son sein dont un président et deux secrétaires. Le bureau est chargé de la gestion courante de la mutuelle de santé. Pour assurer le contrôle et le respect des procédures de gestion, le Conseil d'Administration élit en son sein un Contrôleur Interne. Il a pour rôle le contrôle de toutes les opérations effectuées par le bureau. Les mutuelles d'une même zone utilisant le même hôpital de référence se mettent ensemble pour former un creuset appelé "inter-mutuelle ». L'inter-mutuelle est un niveau d'échanges et de mutualisation des risques lourds comme les interventions chirurgicales en urgence. Elle se réunit en session ordinaire trois fois l'année et assure la représentation des mutuelles dans les négociations avec les hôpitaux. Les mutuelles d'une même commune se mettent ensemble pour créer l'Union Communale des Mutuelles de Santé qui joue le rôle d'interface entre les Mutuelles à la base avec l'administration communale et est un regroupement de mutuelles au niveau local.

Les mutuelles de santé présentes dans le département du Borgou offrent aux populations trois types de services : le produit familial, le produit scolaire et le produit maternité sans risque. Le produit familial regroupe le paquet de soins primaires et les soins hospitaliers offerts aux ménages et constitue le produit général de la mutuelle. La cotisation moyenne pour ce produit est de 2040 FCFA par personne et par an. Quant au produit scolaire, il est composé des soins curatifs ambulatoires et des hospitalisations médicalisées et chirurgicales. Il est offert aux écoliers en cas de survenance du risque à l'école ou lors des activités pédagogiques organisées par l'école, avec une cotisation comprise entre 350 FCFA et 500 FCFA par écolier pour l'année scolaire pour une prise en charge de 100\%. Enfin, le produit maternité sans risques, conçu avant la mise en place de la gratuité des 
césariennes au Bénin couvre les soins de maternité. Pour les accouchements, le mutualiste paye une franchise de 1000 FCFA incluant une couverture à $100 \%$ des consultations prénatales.

Pour être pris en charge dans une des formations sanitaires agréées, le mutualiste doit présenter sa carte d'adhérent en cours de validité. Au départ, cette carte était uniquement détenue par les chefs de ménage, qui pouvaient inscrire comme bénéficiaires les membres de leur ménage. Mais, certains polygynes choisissaient plutôt d'inscrire la première et la dernière épouse, ainsi que les enfants de ces dernières, privant de fait les autres épouses et leurs enfants de la prise en charge médicale en cas de maladie. C'est dans ce contexte que l'adhésion aux mutuelles de santé a été élargie aux femmes, qui peuvent disposer également de la carte d'adhérent.

Les soins de santé pris en charge par l'ensemble des mutuelles sont :

- $\quad$ les maladies ambulatoires : toutes maladies traitées par l’infirmier ou l'infirmière sur place ;

- $\quad$ les consultations prénatales en cas de grossesse ;

- $\quad$ la petite chirurgie : plaie, blessure, coupure, morsures, abcès, panaris, entorse, etc. ;

- $\quad$ les mises en observation ;

- les maladies hospitalisées : paludisme grave, les infections respiratoires aigües, les diarrhées et vomissements ;

- $\quad$ les morsures de serpent et de chien ;

- les interventions chirurgicales en urgence: hernie étranglée, appendicite, césarienne ;

- $\quad$ les accouchements simples ou compliqués et les soins de maternité.

Les médicaments et consommables médicaux utilisés pour les soins mentionnés ci-dessus, sont supportés par la mutuelle à condition qu’ils soient utilisés pour les soins identifiés; qu’ils proviennent des pharmacies régulières des formations sanitaires ; ou qu'ils correspondent aux normes de prescriptions définies par le Ministère de la Santé. Il faut souligner que la mutuelle ne prend pas en charge les prestations lorsque les médicaments sont achetés en dehors de la formation sanitaire ou dans les mains des agents de santé ; tout comme les analyses et les bilans demandés par le malade.

Après les soins, le personnel médical délivre une attestation de soins à chaque mutualiste soigné lorsque ce dernier quitte la formation sanitaire pour rentrer chez lui. Le remboursement des formations sanitaires se fait mensuellement par la mutuelle de santé : c’est le tiers payant. Au niveau de la formation sanitaire une fois les soins administrés, le mutualiste paie sa quotte part qui peut être un ticket modérateur ou une franchise. La prise en charge est comprise entre $75 \%$ et 50\%, c'est-à-dire que le mutualiste paye un ticket modérateur de 25\% à 50\%. Dans les centres de santé, le mutualiste 
paie une franchise comprise entre 600 et 1500 FCFA, les montants au-dessus de cette franchise étant pris en charge par la mutuelle de santé.

\section{La mutuelle de santé : un créneau de sécurisation sociale ?}

Les mutuelles de santé constituent un gage de sécurité sociale pour les populations rurales du département du Borgou. Elles leur confèrent un sentiment de protection et de sérénité par rapport aux craintes liées à la maladie. En effet, l'adhésion à une assurance maladie permet aux mutualistes de bénéficier d'une réduction de 75\% sur les charges liées aux soins de santé. De fait, le sentiment d'inquiétude que suscite la maladie du fait de l'incapacité des populations à mobiliser les ressources financières nécessaires à la couverture des charges médicales se trouve dissipé. Ce point de vue est confirmé par le témoignage d'un chef de ménage de Bonrou dans la commune de Pèrèrè :

"Pour les soins de santé, le ménage mutualiste se sent protégé, sécurisé et possédant de garantie même en l'absence du chef de ménage. En période de gestation où l'automédication est déconseillée chez la femme, on n'a pas de soucis à se faire. A ma connaissance, il n'y a rien de meilleur que la mutuelle de santé ».

L'adhésion aux mutuelles de santé procure donc aux populations rurales du Borgou un sentiment de sécurité, comme l'attestent les propos d'un mutualiste du village de Gah Gbérou dans la commune de Nikki : « je n'ai pas peur lorsque je voyage, car en cas de maladie, les membres de ma famille bénéficient d'une prise en charge du fait de mon adhésion à la mutuelle de santé ». Dans cette perspective, un chef de ménage mutualiste approché à Gbassi dans la commune de Kalalé renchérit : " ma famille est en sécurité lorsque je suis absent». Ce sentiment de sécurité est également reconnu par les femmes comme le prouve le témoignage de Baké, une femme approchée à Fodarou dans la commune de Nikki :

"Depuis que mon mari a adhéré à la mutuelle de santé, je suis beaucoup plus tranquille. Quand mes enfants sont malades, je n'hésite plus à les conduire au centre de santé, parce que les frais que nous payons depuis lors sont désormais très réduits. Avant, ce n'était pas du tout facile parce que mon mari se plaignait souvent de ne pas avoir d'argent pour couvrir les charges médicales. Du coup, nous pratiquions l'automédication. Mais, aujourd'hui la mutuelle de santé nous permet de régler tous ces problèmes et d'être moins stressés, du moins par rapport aux moyens à mobiliser, quand un membre de la famille est malade ».

De fait, il apparaît que la protection apportée par les mutuelles permet au niveau familial de se faire soigner, à un coût réduit, sans pour autant avoir 
besoin de décapitaliser ou de s’endetter. Les mutuelles de santé contribuent ainsi à limiter les risques d’aggravation de la maladie.

En contribuant sensiblement à réduire les charges liées aux soins de santé, les mutuelles de santé permettent aux ménages mutualistes du département du Borgou d'économiser en moyenne près de 50000 FCFA par an (Coopération suisse, 2013). Cette ressource financière non moins négligeable permet d'améliorer les conditions de vie des populations rurales, car elle est utilisée pour soutenir les charges scolaires, alimentaires et vestimentaires du ménage. Elle leur confère au ménage ainsi une certaine tranquillité sociale au regard de son influence sensible sur le bien-être social des populations.

Par ailleurs, l'adhésion aux mutuelles de santé contribuent à réduire sensiblement les dépenses liées aux pratiques parallèles (surfacturation, faux frais, vente de médicaments personnels, détournements de malade, marchandage de privilèges, etc.). Les pratiques dites parallèles, font partie du quotidien des hôpitaux et consistent en une privatisation des soins dans l'espace public hospitalier. Elles sont au cœur des interactions soignantsoigné, mise sur la méconnaissance des circuits officiels d'accès aux services de soins par les usagers ainsi que sur leur situation de détresse. Elles sont classées dans la "petite corruption » généralisée dans les administrations ouest africaine (Blundo et Olivier de Sardan, 2007). Si les pratiques parallèles enrichissent les agents de santé et facilitent la tâche à quelques avertis qui y voient un raccourci d'accès rapide aux soins et à des privilèges, elles plombent néanmoins la qualité des soins et appauvrissent les formations sanitaires. Elles engendrent également l'exclusion d'un grand nombre d'usagers des services de santé. Cette situation explique d'ailleurs selon les entretiens individuels approfondis réalisés l'impopularité des mutualistes auprès de certains agents de santé. La traçabilité des services offerts aux mutualistes ne facilitant pas de telles transactions corruptrices. Sous un autre angle, Criel et al. (2005), ont évoqué le difficile rapport entre professionnels de santé et mutualistes étant donné que ces derniers s'écartent des modes courants de sociabilité soignant -soigné.

En somme, l'adhésion aux mutuelles de santé offre aux populations rurales du Borgou, une certaine protection et une sécurité financière, car elle réduit les paiements directs des ménages dans les formations sanitaires pour les soins primaires, hospitaliers et de maternité. Elle participe aussi à l'amélioration de la gouvernance des systèmes de santé

\section{Les mutuelles de santé : un facteur d'équité sociale ?}

Dans tous les groupes socioculturels étudiés, l'homme est défini comme le responsable de la famille. La femme est perçue comme un être faible placé sous l'autorité et la protection de son époux. Ainsi, des rôles qui 
sont culturellement attribués à la femme ne peuvent être assumés par l'homme, même si elle était malade ou absente. Chez les Peulh, selon la coutume, « la femme est la propriété de l'homme », si bien que lorsqu'elle a des biens matériels et financiers, leur gestion dépend de l'homme qui peut décider de les aliéner ou pas pour les soins de santé. Dans le domaine de la santé, la répartition des rôles sociaux confère la gestion de la santé familiale aux femmes. Elles ont la responsabilité de s’occuper des soins de santé. Les hommes ne s'impliquent que lorsqu'après leurs efforts, la maladie dure dans le temps. Cette répartition des rôles sociaux impliquent pour les femmes des dépenses financières dont elles n’ont pas toujours les moyens: achat de plantes médicinales pour la tisane, achat de médicaments pour baisser la fièvre, réduire des coliques, soigner toux et grippe... Les hommes ne s’occupent que des dépenses hospitalières. L’adhésion à la mutuelle de santé implique la réduction des pratiques d'automédication, et de ce fait, les primo dépenses que font les femmes. Les membres du ménage mutualiste se présentent spontanément à l'hôpital pour leurs soins et les mamans n’attendent plus le dernier moment pour y conduire leurs enfants.

Les mutuelles de santé permettent ainsi de réduire les disparités liées à l'accès des populations rurales du département du Borgou aux soins de santé. Dans cette perspective, elles apparaissent pour les communautés locales comme un moyen de réduction des problèmes liés aux soins de santé. L'inaccessibilité aux centres de santé du fait de l'insuffisance des ressources financières se trouve dès lors remise en cause. L'adaptation des conditions d'adhésion des populations aux réalités sociales du milieu permet aux ménages, même les plus défavorisés, de bénéficier de soins modernes de santé. Les mutuelles de santé ont ainsi contribué à réduire à près de $70 \%$ (Coopération suisse, 2013), les exclusions temporaires ou partielles au niveau des communautés rurales du Borgou et à environ $100 \%$ les exclusions partielles ou temporaires chez les parturientes dont seulement 4,2 \% chez les non adhérentes. Le témoignage d'une femme approchée à Blémi dans la commune de Kalalé confirme la réduction des exclusions :

«Grâce à la mutuelle de santé, le mythe de la fréquentation des centres de santé qu'on croyait réservée uniquement à ceux qui ont de l'argent se trouve aujourd'hui brisé. Malgré les maigres ressources que j'ai, je peux aujourd'hui me rendre dans un hôpital pour me soigner ou pour soigner les membres de ma famille sans faire de grosses dépenses ; ce qui n'était pas le cas avant la mise en place des mutuelles dans la localité ».

Les mutuelles de santé offrent donc aux ménages, même les plus défavorisés, la possibilité de bénéficier de soins de santé modernes et de qualité. Ce faisant, elles constituent un instrument de réduction de la différenciation sociale. Une femme non adhérente s’exprime comme suit : « 
quand vous êtes mutualiste et que vous accouchez à l'hôpital, on vous libère le même jour, c'est une fierté. Alors qu'il arrive que les non-mutualistes soient gardés parce que le payement des frais d'hospitalisation se fait souvent attendre ». Cette différence dans le traitement des patients est perçue comme un privilège social pour les communautés locales. C'est d'ailleurs à juste titre que Bio, un sage du village de Gah Gbérou dans la commune de Nikki soutient que «dans le village ici, être mutualiste constitue aussi une fierté ».

L'adhésion à la mutualité permet par exemple au chef de ménage de disposer d'un meilleur statut social. En effet, chaque chef de ménage quel que soit son rang social à la possibilité d'offrir aux membres de sa famille des soins modernes de santé. Au même titre que les grands cotonculteurs, les petits producteurs de coton détenteurs d'une carte d'adhérents peuvent ainsi bénéficier des mêmes droits de soins.

L'adhésion aux mutuelles de santé augmente la liberté d'expression des patients à l'égard des agents de santé. Dans cette perspective, un agriculteur à Nikki affirme :

«Les agents de santé ont fait la pluie et le beau temps dans ce village. Ils étaient tellement arrogants qu'il était parfois difficile aux malades de leur décrire correctement leurs maux. Mais depuis mon adhésion à la mutuelle, j'ai plus de facilité à échanger librement avec le personnel médical ».

\section{Les mutuelles de santé, un instrument de transformation des rôles de genre}

Si les descriptions précédentes ont mis en exergue les bénéfices des mutuelles sur l'accès à la santé et les solidarités communautaires, les données collectées font également montre d'une autre fonction des mutuelles de santé. Celle de catalyseur des rapports hommes femmes en matière de gestion de la santé. Avec les mutuelles de santé, les hommes autrefois absents des processus d'accès aux soins de santé des femmes et des enfants, dans les centres de santé, se retrouvent en amont et en aval du fait de leurs appartenance aux mutuelles. L’adhésion aux mutuelles implique un certain engagement, une participation aux réunions d’informations sur le système de soins et une responsabilisation du chef de ménage (souvent le père de famille). Ainsi non seulement les hommes payent les frais d'adhésion pour leur famille mais aussi contrôlent l'accès effectif aux soins et la garantie de soins de qualité. Valorisés à travers ce nouveau rôle dans gestion de la santé familiale, les hommes deviennent des acteurs du bon fonctionnement et de la promotion des mutuelles mais surtout des intermédiaires clés entre les agents de santé et les usagers des centres de santé (le plus souvent les femmes et les enfants) pour des prestations de qualité. La mise en place des mutuelles de 
santé dans le département du Borgou a ainsi contribué à déconstruire la répartition des rôles sociaux. A titre d'illustration, malgré l'existence de croyances contraignantes non favorable à une remise en cause des rôles, l'avènement des mutuelles de santé opèrent des transformations révolutionnaires. Ainsi chez les Boo par exemple, l'homme n’accompagne pas son ou ses épouse (s) et ses enfants à l'hôpital de peur d'aggraver la maladie.

«Si je n'arrivais pas à accompagner ma femme au centre de santé, c'est parce que chez nous, c'est mal vu. C'est une voisine ou une parente qui garde la femme. On pense que l'homme doit aller travailler. Mais depuis notre adhésion à la mutuelle de santé et suite aux différentes séances de sensibilisations que nous avons eu, je n'hésite pas à conduire moi-même les membres de ma famille à l'hôpital quand ils sont malades».

C'est une manière pour ce mutualiste, de s'assurer de l'effectivité des soins dus en lien avec sa cotisation. Il convient de souligner que cette situation est relativement similaire chez les Bariba où la femme n’accompagne pas son premier fils à l'hôpital. Si elle le fait, c'est le fils qui va en pâtir soit par aggravation de la maladie, soit par la mort. Dans ce sens, autrefois, pendant que les hommes s’occupaient des dépenses des soins sanitaires dès l'intervention d'un agent de santé, ce sont leurs épouses qui s'occupaient des autres aspects de la prise en charge sanitaire (garde, alimentation, hygiène, réconfort, ...). Cette tendance tend cependant à changer grâce aux mutuelles de santé avec une meilleure responsabilisation des hommes quant à la répartition des rôles en matière de prise en charge médicale. De fait, il en découle un glissement des frontières quant aux rôles sociaux assignés aux hommes et aux femmes dans les ménages. Il convient néanmoins de préciser que quel que soit le système culturel considéré dans la zone d'intervention du projet, certaines activités et rôles sont exclusivement du ressort des femmes. Il s’agit de la restauration et de la garde (à domicile ou en hospitalisation). Toutefois, la quasi-totalité des femmes enquêtées évoquent leur contribution aux dépenses de santé de la famille et leur assistance financière à leurs époux en cas de défaillance de ces derniers pour être à jour des cotisations. Cette assistance est permanente, mais moins visible en ce qui concerne les dépenses fréquentes pour l'achat de produits pharmaceutiques sur le marché informel pour soigner les "petites maladies". La contribution des femmes est systématique et plus substantielle lorsqu'elles exercent des activités lucratives en permanence. Dans ce sens, un homme affirme :

«Ma femme m'aide beaucoup dans la résolution des problèmes de santé au niveau du ménage. Après les travaux domestiques, elle met sa bassine sur la tête pour aller chercher de l'igname pour revendre 
en détail. C'est dans cet argent, qu'elle puise pour acheter des comprimés pour ses enfants, m'aide pour des ordonnances éventuelles, m'aide à cotiser pour la mutuelle et achète de viande qu'elle prépare pour tout le monde dans le ménage ».

L'opérationnalisation des mutuelles de santé a ainsi permis d'offrir à des populations rurales déshéritées plus de justice sociale et d'équité genre dans l'accès à des soins de santé de qualité.

\section{Discussion}

La mise en place des mutuelles de santé a contribué à améliorer l'accessibilité des populations rurales du département du Borgou aux soins de santé. Malgré le potentiel de ce département dans la production cotonnière, les populations n'avaient pas toujours un accès facile aux centres de santé, du fait de la forte responsabilisation des seules femmes face au recours de soins dans les formations sanitaires. A partir du développement ci-dessus, il ressort que les mutuelles de santé, constituent pour les populations rurales étudiées un modèle d'accès aux soins de santé, une passerelle pour l'amélioration des dysfonctionnements du système de soins et un creuset de participation communautaire et d'engagement citoyen autour des questions de santé. A titre d'exemple, la mutuelle de Nikki constitue un bel exemple de réduction de la pauvreté à travers l'amélioration de l'accessibilité financière des pauvres et personnes vulnérables aux soins et services de santé de qualité, comme l'attestent ces statistiques : 2000 bénéficiaires pour la garantie familiale, 5000 enfants couverts pour la garantie scolaire, 200 zémidjans couverts pour les risques liés aux accidents de travail, un accroissement de $70 \%$ des grossesses suivies.

L'opérationnalisation des mutuelles de santé a ainsi permis de réduire de nombreuses disparités en matière d'accès aux soins de santé. En mettant en place un système basé sur la solidarité et l'entraide, les mutuelles de santé ont permis à des personnes issues de milieux ruraux déshérités de bénéficier d'une protection sociale. Au regard de l'importance de la notion d'entraide et de solidarité, les systèmes de mutualisation des risques maladie sont classés selon Waelkens et Criel (2004) parmi un large éventail de mécanismes d'entraide qui vont de l'entraide familiale à la sécurité sociale organisée par l’État. A cet effet les auteurs distinguent quatre niveaux de solidarité : la solidarité familiale restreinte et obligatoire ; la réciprocité équilibrée des associations informelles d'entraide qui assurent des bénéfices égaux pour chaque membre ; la solidarité redistributive entre malades et non malades des systèmes d'assurance et la solidarité verticale entre riches et pauvres qui existe dans les systèmes européens de sécurité sociale. Il apparaît donc que les mutuelles de santé constituent dans leur globalité, une forme 
traditionnelle d'organisation d'économie sociale et solidaire. Les pratiques d'économie solidaire :

«Privilégient la recherche de la solidarité (entre générations et avec les générations futures, entre territoires, entre riches et pauvres, entre sexes, etc.). Elles ne cherchent pas à supplanter le marché ou l'Etat, mais plutôt à réarticuler ces deux institutions avec les principes de réciprocité et de solidarité. Elles inventent ou réinventent des formes de gestion non capitalistes qui permettent la réappropriation des moyens de production. Elles expérimentent aussi un nouveau rapport au politique, en articulant leurs pratiques économiques avec la création d'espaces de délibération et de débat public et avec la volonté de peser sur la définition et la mise en œuvre des politiques publiques et sur les normes dominantes de l'économie de marché et des institutions » (Verschuur et al., 2014).

L'organisation de cette forme de protection sociale contribue de façon générale à assurer une sécurité de revenu et à protéger les ménages des risques sociaux tels que la maladie, l'invalidité, les accidents du travail et les maladies professionnelles, le chômage, la vieillesse ou le décès. Pour les populations rurales du département du Borgou, elle leur permet de jouir d'une certaine quiétude sociale.

La promotion de la protection sociale apparaît pour le Bureau International du Travail, et particulièrement son programme Stratégies et Techniques contre l'Exclusion sociale et la Pauvreté (BIT/STEP) comme l'expression d'une vision de la société.

«La sécurité sociale est un droit fondamental de l'être humain et un instrument essentiel de cohésion sociale, qui par là même concourt à la paix et à l'insertion sociale. Composante indispensable de la politique sociale, elle joue un rôle capital dans la prévention et la lutte contre la pauvreté. En favorisant la solidarité nationale et le partage équitable des charges, la sécurité sociale peut contribuer à la dignité humaine, à l'équité et à la justice sociale. Elle est importante également pour l'intégration, la participation des citoyens et le développement de la démocratie. Si elle est bien gérée, la sécurité sociale favorise la productivité en assurant des soins de santé, une sécurité du revenu et des services sociaux. Associée à une économie en expansion et à des politiques actives du marché du travail, elle est un instrument de développement économique et social durable » (BIT, 2002).

Pour les populations rurales approchées, les mutuelles de santé leur permettent de jouir de cette sécurité sociale, car ils peuvent désormais vaquer à leurs occupations sans trop s’inquiéter des soins de santé des membres de leurs familles. Pour les femmes et autres personnes déshéritées, l'adhésion à 
la mutuelle constitue un moyen de vaincre la discrimination sociale. « Grâce à la mutuelle, je n'ai plus besoin d'attendre l'aide de mon mari pour mieux prendre en charge ma santé et celle de mes enfants ». Ces propos sont renchéris par ceux d'un homme. «Sans la mutuelle, je ne pourrais par offrir aux membres de ma famille des soins de qualité ». Les mutuelles de santé se révèlent ainsi être des éléments de promotion de justice sociale en ce sens qu'elles offrent à toutes les catégories sociales les mêmes chances d'accès à des soins de santé modernes.

Cependant, si les prestataires comprennent l'intérêt d'une mutuelle comme un instrument technique de prépaiement des soins, ils ne la perçoivent pas encore comme un instrument de promotion de la qualité des soins, dans l'intérêt de ses adhérents (Waelkens, Criel, 2004). Selon Fournier (1995), la connaissance de la mutuelle de santé à travers ses principes et les prestations qu'elle offre, peut inciter les personnes à souscrire ou non au système de prépaiement de leurs soins de santé et cela en fonction de leur capacité financière qui dépend des sources de revenu, et de l'activité génératrice de revenu et des capacités à épargner des personnes. La prise de conscience du risque de maladie est également un facteur déterminant qui peut susciter le désir de s'assurer sur le plan de la santé avec le système de prépaiement de la mutualité. Une fois cette prise de conscience réelle et l'adhésion effective, la mutuelle assure ce risque éventuel. La maladie une fois manifeste, engendre des besoins de soins qui lorsqu'ils sont pris en compte parmi les prestations de la mutuelle seront couverts. Après la satisfaction des besoins de soins, les populations peuvent accroître leur capacité productive et donc améliorer leur capacité financière. Toutefois, ces relations ne sont pas nécessairement exhaustives dans la mesure où elles peuvent être traduites par d'autres facteurs implicites : mauvais accueil, barrières socioculturelles, stratégies de chaque promoteur de Mutuelle de Santé et qui peuvent cependant influer sur la faible mutualisation des communautés (Fournier, op. cit.). Les mutuelles de santé apparaissent de fait comme une véritable stratégie visant à promouvoir l'équité sociale dans le département du Borgou au Bénin. C'est fort de ce potentiel que le Gouvernement du Bénin s'inspirant des mutuelles de santé a initié le Régime d'Assurance Maladie Universelle (RAMU) en vue de protéger l'ensemble des populations béninoises contre les conséquences financières du risque maladie. C'est un dispositif destiné à assurer une meilleure coordination et un fonctionnement harmonieux et efficient des mécanismes déjà disponibles, à créer un mécanisme d'insertion des travailleurs des secteurs informel et agricole ainsi que des couches sociales démunies dans le dispositif de prise en charge, tout en veillant au respect des principes de base que sont la couverture universelle, l'approche contributive, la solidarité nationale, la 
responsabilité générale de l'Etat, l’équité, la gestion participative et l'efficience.

Toutefois, au-delà de ces fonctions incontestées des mutuelles de santé, apparait celle non moins importante de transformation et de redistribution des rôles des hommes et des femmes dans la gestion des soins de santé familiale. Dans une société majoritairement constituée de Bariba, de Peulh et de Boo, dans laquelle culturellement l'homme ne conduit pas sa femme à l'hôpital, elles contribuent à déconstruire les rôles sociaux. Les hommes conduisent désormais avec fierté leurs épouses au centre de santé et interviennent dans le financement prépayé des soins. Leur absence du domicile conjugal n'est plus un frein au recours aux soins du reste de la famille. Les femmes ne font plus seules face aux dépenses en l'absence du chef de famille lors des urgences médicales. Bénéficiant des services de promotion de la santé offerts aux mutualistes, elles ne sont plus seules dans la prévention. Les mutuelles de santé permettent à tous les bénéficiaires hommes et femmes d'être mieux informés au sujet des problèmes d'accès aux soins, des soins délivrés par les formations sanitaires et de leurs coûts. Plus sûrs de leur bon droit, ils peuvent se montrer plus exigeants et exprimer leur point de vue avec davantage de force (Criel et al. 2006). En outre on observe toujours avec l'avènement des mutuelles que la contribution des femmes souvent invisible dans la quête de tisanes, de médicaments de la rue, etc., prend un caractère officiel et visible avec l'adhésion directe aux mutuelles et la participation aux cotisations ou le payement direct du ticket modérateur. Les femmes ont davantage ainsi la possibilité de contribuer aux soins de santé des membres de leurs familles de façon tangible et reconnue.

\section{Conclusion}

Cette recherche analyse les effets des mutuelles de santé sur l'accès aux soins équitable des populations dans le département du Borgou au Bénin. Elle a surtout rendu compte des mutations qu'elles induisent dans la division sexuelles des rôles de genre autour de la gestion familiale de la santé. Dans un premier temps, une brève historique des mutuelles de santé a été présentée ainsi qu'une description de leur mode de fonctionnement. A cet effet, il ressort que si les premières mutuelles de santé sont apparues au Bénin en 1990, c'est en 1994 qu'elles ont été mises en œuvre dans le département du Borgou grâce à l'appui financier de la Coopération Suisse. Aujourd'hui, le département compte 31 mutuelles de santé Qui offrent un paquet de soins préventifs et curatifs à plus de 20000 adhérents. Elles contribuent à sécuriser les ménages en matière de santé. En effet, l'adhésion aux mutuelles de santé permet aux mutualistes d'avoir un sentiment de quiétude par rapport aux risques liée à leur santé et à celle des membres de leurs familles. Certains chefs de ménages voyagent ainsi en toute tranquillité 
confiant du fait que même en cas de maladie, la carte d'adhérent permettrait d'assurer à leur famille une prise en charge médicale. Les craintes et les peurs liées aux soins de santé familiale se trouvent de fait dissiper. Les mutuelles offrent donc aux populations rurales une garantie en matière de prise en charge de leur santé. En diminuant sensiblement les charges financières liées aux soins de santé, les mutuelles de santé offrent aux populations rurales du bastion cotonnier du Bénin, la possibilité de réduire de $70 \%$ les exclusions temporaires ou partielles des communautés rurales aux centres de santé et à $100 \%$ les exclusions temporaires ou partielles des parturientes.

Par ailleurs, elles contribuent également à déconstruire les rapports sociaux de sexe dans la gestion de la santé familiale en milieux peulh, boo et bariba Les hommes s'impliquent davantage dans la prise en charge sanitaire des membres de la famille et le rôle des femmes apparaît plus visible. Contrairement aux pratiques traditionnelles, ils conduisent femmes et enfants aux centres de santé, sont présents lors des consultations et assurent le suivi d’application des prescriptions médicales à la maison. Le produit maternité sans risque agit non seulement sur la systématisation des consultations pré et post natales, favorisant un meilleur suivi des grossesses et réduisant les complications et les risques de mortalité maternelle et infantile. Cependant, il est important de s'interroger sur l'influence des mutualités sur la qualité des soins de santé au Bénin et plus particulièrement dans le département du Borgou. Mais si les mutuelles de santé dans ce département servent d’échelle pour l'accès aux soins de santé des populations vulnérables et instaurent une justice sociale dans la gestion de la santé familiale, leur durabilité est souvent mise à l'épreuve en l'absence d'une politique nationale stable d'assurance maladie au-delà de tout changement de gouvernement.

\section{References:}

1. Aladji Boni Sylla, A-R. D. (nd). Recueil de sept histoires insolites relevant du domaine de la santé et du développement des mutuelles de santé.

2. BIT (2002). Sécurité sociale : un nouveau consensus. Genève : Bureau International du Travail.

3. Blundo, G. et J.-P. Olivier de Sardan (éds.) (2007). Etat et corruption en Afrique. Une anthropologie comparative des relations entre fonctionnaires et usagers (Bénin, Niger, Sénégal). Paris : APADKarthala.

4. Coopération suisse (2013). Capitalisation de dix-neuf ans d'expérience d'appui de la Coopération Suisse aux mutuelles de santé dans les départements du Borgou et des Collines au Nord Bénin. 
5. Criel B., Blaise, P. et Ferette, D. (2006). Mutuelles de santé en Afrique et qualité des soins dans les services : Une interaction dynamique. L'Assurance maladie en Afrique francophone Améliorer l'accès aux soins et lutter contre la pauvreté. Banque Mondiale, Washington, pp 353-371.

6. Criel, B., Ahmadou Diallo, A., Van der Vennet, J., Waelkens, M-P. et Wiegandt, M. (2005). La difficulté du partenariat entre professionnels de santé et mutualistes: le cas de la mutuelle de santé Maliando en Guinée-Conakry. Tropical Medicine \& International Health, 10(5), pp. 450-463, http://onlinelibrary.wiley.com/doi/10.1111/j.13653156.2005.01410.x/full, consulté le 23 mars 2016.

7. Defourny, J. et Failon, J. (2011). Les déterminants de l'adhésion aux mutuelles de santé en Afrique subsaharienne: un inventaire des travaux empiriques. Mondes en développement, (153), pp. 726, www.cairn.info/revue-mondes-en-developpement-2011-1-page-7.htm, consulté le 24 mars 2016.

8. Doumbouya, M. L. (2008). Accessibilité des services de santé en Afrique de l'Ouest : le cas de la Guinée. Working paper, (2), https://halshs.archives-ouvertes.fr/halshs-00229696/document, consulté le 23 mars 2016.

9. Fournier P, Haddad, (1995). Les facteurs associés à l'utilisation des services de santé dans les pays en développement. Montréal (Canada) : éd Gérard H.

10. Ministère de la Santé du Bénin, (2007). Plan Stratégique de Développement des Mutuelles de Santé 2007-2011.

11. Parini, L, (2006). Le système genre. Introduction aux concepts et théories. Editions Seismo, Zürich, pp 40-52.

12. Scott, J-W (2000). Genre : une catégorie utile d'analyse historique. In Bisilliat, J., Verschuur et C.C (Dir.) : Le genre : un outil nécessaire. Introduction à une problématique. Cahiers genre et développement $n^{\circ} 1$. Paris : L’Harmattan, pp 41-67.

13. Verschuur, C., Guerin, I, Guétat-Bernard, H., (2014), Under Development: Gender, Palgrave Macmillan.

14. Vidal, C, (2008). Le cerveau a-t-il un sexe ? Direction générale de l'enseignement scolaire. .

15. Waelkens, M-P. et Criel, B. (2004). Les mutuelles de santé en Afrique subsaharienne. État des Lieux et Réflexions sur un Agenda de Recherche. Health, Nutrition and Population (HNP) Discussion Paper, http://documents.banquemondiale.org/curated/fr/2004/03/4125189/le s-mutuelles-de-sante-en-afrique-sub-saharienne-etat-des-lieuxreflexions-sur-un-agenda-de-recherche, consulté le 23 mars 2016. 